\title{
El aprendizaje activo en traducción y su evaluación
}

\author{
PiLAR ELENA \\ Universidad de Salamanca \\ pel@usal.es
}

Recibido: 10 de noviembre de 2010

Aceptado: 12 de diciembre de 2010

\section{RESUMEN}

La adaptación de los títulos universitarios al EEES y la consiguiente adopción del sistema de créditos ECTS supone el inicio de una nueva etapa educativa que implica cambios en la tradicional forma de concebir el proceso enseñanza-aprendizaje. El peso del tradicional enseñar se desplaza a la noción de aprender: el profesor deja de ser en un mero transmisor de conocimientos para adoptar el papel de asesor y guía en el proceso de aprendizaje del alumno. Este cambio en el esquema didáctico comporta profundas adaptaciones para ambos que se reflejan en la relación entre ellos, en el papel que representan en el nuevo marco pedagógico, en la organización de las materias, en los objetivos y en los métodos. También en los sistemas de evaluación, ya que ésta adquiere un nuevo protagonismo desde la perspectiva del aprendizaje activo.

Palabras clave: métodos activos en la enseñanza, tipos de evaluación, didáctica de la traducción.

\section{Active Learning and Assessment in Translation}

\begin{abstract}
With the adaptation of higher education degrees to the EEES and the subsequent application of the ECTS credit system, university education is embarking on a new path with major changes in the traditional teaching-learning process. The traditional emphasis on teaching is increasingly outweighed by a stress on learning: teachers are no longer mere transmitters of knowledge but have become facilitators and guides in the learning process. These changes in the new pedagogical framework entail major adaptations in the relationship between students and teachers, particularly affecting each one's role in the process as well as the organization, objectives and methodologies used on courses. Such changes also affect assessment, which now takes on a central role in all active learning frameworks.
\end{abstract}

Key words: Active teaching methods, types of assessment, translation didactics 
Sumario: 0. Introducción. 1. Competencias y planificación docente. 1.1. Las metodologías activas. 2. La evaluación. 2.1. La evaluación alternativa. 2.1.1. La carpeta de aprendizaje. 3. Conclusiones.

\section{O. Introducción}

El nuevo sistema educativo se propone conseguir un doble objetivo: por una parte, acercar la universidad a la sociedad y al ámbito laboral y, por otra, ofrecer al estudiante una enseñanza más adecuada a su futuro mundo profesional. Esta nueva orientación conlleva adaptaciones importantes tanto para el profesor como para el alumno que se reflejan en los nuevos planes de estudio en los que se establece la base de la enseñanza-aprendizaje en el conjunto de competencias que una persona debe poseer para poder ejercer su función laboral dentro de la sociedad. La noción de competencia ha sido ya tratada en numerosos estudios ${ }^{1}$, en términos generales se dice de la competencia que es el conjunto de conocimientos, actitudes y destrezas que habilitan para el ejercicio de una profesión. El Ministerio de Educación y Cultura $(2006)^{2}$ define el término de la siguiente manera: "competencia es una combinación de conocimientos, habilidades (intelectuales, manuales, sociales, etc.), actitudes y valores que capacitarán a un titulado para afrontar con garantías la resolución de problemas o la intervención en un asunto en un contexto académico, profesional o social determinado". En ésta como en otras definiciones se destaca el hecho de que para ejercer una función dada $^{3}$ el profesional competente no sólo debe poseer los conocimientos necesarios, saber, sino que son además imprescindibles unas determinadas habilidades, saber hacer, y unas actitudes, saber estar/ser (en la pirámide de Miller, saber actuar); es decir, todo un conjunto de recursos que tendrá que ser utilizado eficazmente para conseguir un resultado definido en un entorno laboral dado y en diferentes situaciones (Le Boterf 2001).

\section{Competencias y planificación docente}

Existen diversos tipos de competencias, competencias básicas que una formación universitaria debe garantizar, tal y como se recoge en el Real Decreto 1393/2007, y otras transversales y específicas establecidas en cada titulación de grado $^{4}$. A efectos didácticos parece oportuno agrupar la larga lista de éstas en una

\footnotetext{
1 Sobre el concepto y clases de competencia vid. Yániz 2008, Villardón 2006, Pérez Pueyo et. Al. 2008. En el campo de la traducción: Hurtado 2001, Orozco/Hurtado 2001, PACTE 2001, Kelly 2005, Galán 2007 y Galán 2009.

${ }^{2}$ En la propuesta sobre las Directrices para la elaboración de Títulos Universitarios de Grado y Máster.

3 También sería posible enfocar esta cuestión desde la perspectiva contraria, y definir competencia como el conjunto de tareas que requiere un determinado puesto de trabajo para su desempeño, en este caso los criterios que debe cumplir el producto elaborado por un profesional pueden convertirse en el paradigma de los objetivos finales de la formación.

4 Las competencias transversales y específicas del Grado en Traducción e Interpretación de la Universidad de Salamanca se pueden consultar en www.usal.es.
} 
serie de categorías competenciales universales, válidas para cualquier ámbito ${ }^{5}$, como son la competencia técnica, la competencia metodológica y la competencia profesional, que servirán de guía en la organización de la actividad docente:

\begin{tabular}{|c|c|c|}
\hline Categorías competenciales & Contenido & Competencia traductora \\
\hline $\begin{array}{l}\text { Competencia técnica }(\mathrm{CT}) \\
\text { (Saber) }\end{array}$ & $\begin{array}{l}\text { Es el dominio experto de las tareas y } \\
\text { contenidos del ámbito del trabajo, } \\
\text { así como los conocimientos y } \\
\text { destrezas necesarios para ello. } \\
\text { Comprensión sistémica y gestión } \\
\text { de información }\end{array}$ & $\begin{array}{l}\text { Conocimientos textuales } \\
\text { (incl. lingüísticos y } \\
\text { extralingüísticos). } \\
\text { Conocimientos de la profesión. }\end{array}$ \\
\hline $\begin{array}{l}\text { Competencia } \\
\text { metodológica }(\mathrm{CM}) \\
\text { (Saber hacer) }\end{array}$ & $\begin{array}{l}\text { Aplicación del procedimiento adecuado } \\
\text { a las tareas encomendadas y a los } \\
\text { problemas que se presenten, } \\
\text { búsqueda de soluciones y transferencia } \\
\text { de las experiencias a las nuevas } \\
\text { situaciones } \\
\text { de trabajo. Gestión de información y } \\
\text { dominio tecnológico }\end{array}$ & $\begin{array}{l}\text { Capacidad para aplicar los } \\
\text { conocimientos al trabajo } \\
\text { de análisis, transferencia y } \\
\text { revisión de textos. } \\
\text { Capacidad documental y } \\
\text { tecnológica. }\end{array}$ \\
\hline $\begin{array}{l}\text { Competencia participativa } \\
\text { (saber estar) y competencia } \\
\text { personal (saber ser) }= \\
\text { Competencia profesional } \\
\text { (CP) }\end{array}$ & $\begin{array}{l}\text { Capacidad de organizar, decidir y } \\
\text { aceptar responsabilidades. } \\
\text { Gestión de recursos y relaciones } \\
\text { interpersonales. } \\
\text { Comportamiento orientado al grupo } \\
\text { y al entendimiento interpersonal. } \\
\text { Relaciones interpersonales }\end{array}$ & $\begin{array}{l}\text { Capacidad de gestionar } \\
\text { proyectos de traducción } \\
\text { en un entorno laboral real. } \\
\text { Capacidad de trabajo en } \\
\text { equipo, de relacionarse con } \\
\text { los demás de una forma eficaz }\end{array}$ \\
\hline
\end{tabular}

La planificación docente corre a cargo del profesor que tiene ante sí el reto de decidir sobre dos cuestiones que influirán decisivamente en la formación del estudiante: cómo organizar una asignatura para que ofrezca la posibilidad de adquirir las competencias requeridas y cómo lograr que el alumno aprenda de forma autónoma, reflexiva y crítica. Para lograr este doble objetivo son cuatro las áreas de actuación disponibles: los contenidos, los objetivos, la metodología y la evaluación. Cada uno de estos elementos se interrelaciona con el resto y todos ellos están marcados por las competencias establecidas para cada titulación. La innovación docente, necesaria para hacer frente a los nuevos contenidos y objetivos de aprendizaje, obliga al empleo de metodologías activas, combinadas con las tradicionales, de tal forma que permitan al alumno descubrir y construir conocimientos por ellos mismos (el cons-

5 En el campo de la traducción el modelo competencial de PACTE (2001) contempla las siguientes categorías: competencia lingüística, competencia extralingüística, competencia de transferencia, competencia instrumental-profesional, competencia psicofisiológica y competencia estratégica. 
tructivismo de Piaget), al mismo tiempo que fomenten su actitud positiva hacia el aprendizaje. En general, son cinco los factores decisivos en el diseño del conjunto de la actividad docente:

a) el nivel de desarrollo de la actividad educativa (grado (curso), máster),

b) los contenidos que se pretenden ofrecer (técnicos, metodológicos, profesionales),

c) los tipos de actividad de aprendizaje: metodologías tradicionales (clases magistrales, seminarios) o metodologías activas,

d) los tipos de relación enseñanza-aprendizaje (expositiva directa, dirigida, asistida, autónoma, en cooperación con otros estudiantes) y

e) el tipo de evaluación, instrumentos evaluativos y criterios.

\subsection{Las metodologias activas}

Las metodologías activas tienen como objetivo asegurar que el estudiante comparte activamente la responsabilidad en su proceso de aprendizaje y además que se prepara para aprender autónomamente o en equipo a lo largo de su vida. Entre las estrategias activas se encuentran: el aprendizaje colaborativo (AC), el aprendizaje basado en problemas (ABP) y el método de casos (MC), también la carpeta de aprendizaje (CA).

El AC es un método basado en el constructivismo social de Vigotsky que postula que sólo en un contexto social se logra aprendizaje significativo; se trata de la realización de trabajos en grupo en los que cada integrante del equipo tiene una tarea encomendada ${ }^{6}$. Según Barkley et al. (2007) el AC consiste en que dos o más estudiantes trabajen juntos y compartan equitativamente la carga de trabajo mientras progresan hacia los resultados de aprendizaje previstos.

La metodología del ABP tiene sus orígenes en los estudios de Medicina de la Universidad de MacMaster (Canadá) ${ }^{7}$. Este método se centra en la investigación y reflexión que sigue el alumno para llegar a una solución ante un problema planteado por el profesor. Los protagonistas del aprendizaje son los alumnos que asumen la responsabilidad de ser parte activa del proceso de aprendizaje.

El método de casos (MC) consiste en proponer un "caso" a un grupo de alumnos para que individualmente o colectivamente lo sometan a análisis y tomen decisiones (Berná 2006). Con ello se pretende que los alumnos analicen la situación, aprendan a definir los problemas, tomen decisiones acerca de la búsqueda de soluciones, contrasten ideas y las reelaboren con nuevas aportaciones.

Estos tres métodos tienen en común varios factores:

- el aprendizaje está centrado en el alumno,

\footnotetext{
${ }^{6}$ Para más información sobre el aprendizaje colaborativo en traducción vid. Kiraly 2005, Tortadès 2006 y Echeverri 2005.

${ }^{7}$ Sobre el aprendizaje basado en problemas vid. Morales y Landa 2004, Ramírez 2005 y Font 2003.
} 
- el aprendizaje se produce en pequeños grupos,

- los profesores son asesores o guías.

También comparten varias condiciones:

- el profesor debe diseñar las actividades, a veces esta tarea la comparte con el alumno, con unos objetivos claramente definidos,

- todos los participantes deben colaborar en las tareas encomendadas,

- el aprendizaje debe ser significativo, constructivo, no memorístico.

\section{La evaluación}

La evaluación ${ }^{8}$ constituye en el actual marco pedagógico un aspecto fundamental tanto para el proceso de la formación como para su resultado. Dejando de lado las consecuencias negativas que determinadas formas de evaluar hayan tenido en la formación del alumno (McDonald 2000), hoy se plantean "aproximaciones evaluativas que promueven el aprendizaje del estudiante, dentro de la cultura de la evaluación para el aprendizaje" (Villardón 2006: 59). Se abre un nuevo rumbo en el enfoque de la función evaluativa que pasa de esta manera a formar parte del aprendizaje como una faceta más de éste. Esto significa que se convierte en una pieza fundamental del aprendizaje activo en el que el estudiante debe aprender a evaluar y debe evaluar para aprender. Esta forma de evaluación, alejada de la tradicional calificación del resultado de aprendizaje como única alternativa, supone replantearse dos aspectos fundamentales (Delgado, A. et al. 2005):

a) la determinación de los tipos y de las actividades concretas de evaluación y

b) la información al estudiante, clara y precisa, sobre objetivos, tipos de evaluación, y criterios de evaluación y de calificación.

Por lo que respecta al primer aspecto es conveniente plantearse una serie de reflexiones (Villardón 2006: 62):

- Si la competencia es la suma de conocimientos, habilidades y actitudes, la evaluación debería abarcar estas tres adquisiciones.

- Si la competencia supone la movilización estratégica de estos tres recursos para dar respuesta a una situación determinada, la evaluación debe constatar si esta capacidad se ha desarrollado de forma eficaz y ética.

- Si el desarrollo de competencias es un proceso de aprendizaje, se habría de valorar este proceso, lo cual redundará en beneficio de los objetivos formativos.

En relación al segundo aspecto, dos de las cuestiones que demandan más información por parte del alumno son la organización de las actividades y tareas, y los

8 Aquí nos referiremos únicamente a la evaluación en el proceso de aprendizaje, existen otros aspectos (Martínez Menlis, N. y Hurtado 2001), como la crítica o valoración de traducciones (Elena 1998) y la revisión de traducciones (Parra 2007 y 2009) en el ámbito profesional. 
tipos y criterios de evaluación. Una buena estrategia de comunicación (Blanch 2009 y Berná 2008) es la que prevé el contenido de la información y el momento en el que ésta se proporciona. Teniendo en cuenta estas consideraciones previas, la base para un buen diseño del sistema de evaluación se fundamenta en tres principios evaluativos generales (McDonald et al. 2000):

- Usar métodos de evaluación adecuados que permitan evaluar la competencia de manera integrada con sus criterios de realización.

- Utilizar métodos que sean directos y relevantes para lo que se está evaluando.

- Basarse en una amplia gama de pruebas para valorar la competencia.

Según la finalidad y el momento de realización existen tres tipos de evaluación: evaluación diagnóstica o inicial, evaluación formativa o continua y evaluación sumativa o final (Orozco 2006, Galán 2007 y Galán 2009).

La evaluación diagnóstica o inicial es la que se realiza antes de empezar el proceso de aprendizaje (o al comienzo de una parte o un tema), este tipo de evaluación pretende ajustar el punto de partida de la formación a los niveles reales de conocimientos y habilidades de los estudiantes con el fin de elegir la secuencia formativa que mejor se adapte al nivel. Debe detectar los aprendizajes adquiridos y también las lagunas. Los instrumentos que se emplean para una evaluación diagnóstica son variados, puede realizarse por escrito, a través de una prueba o un cuestionario, o bien a través de la observación de la reacción de los estudiantes ante una explicación. Se trata de obtener por medio de instrumentos adecuados información sobre competencias técnicas, metodológicas y profesionales ya existentes.

La evaluación formativa o continua favorece el logro del aprendizaje de competencias o elementos de éstas. Es la evaluación centrada en el proceso del aprendizaje, por tanto la participación activa del estudiante es básica. La evaluación formativa es también continua, consiste, básicamente, en proponer al estudiante una serie de actividades que deberá ir realizando a lo largo del curso con la doble finalidad de obtener datos, tanto el profesor como el estudiante, acerca del proceso formativo (para modificar, mejorar, pautar el ritmo de trabajo de los estudiantes) y evaluar su proceso de aprendizaje. Una parte importante de la tarea del profesor en un método de evaluación formativa-continua es la corrección de las actividades que realiza el estudiante y la comunicación de los resultados de la corrección. El éxito de la evaluación continua es directamente proporcional a la personalización del retorno que el estudiante recibe del profesor sobre el trabajo que ha realizado. Las condiciones de este tipo de evaluación son, por tanto, fundamentalmente dos:

- Debe realizarse durante el proceso de formación para que la mejora pueda formar parte de ese proceso.

- Debe proporcionar una retroinformación (retroalimentación, retorno, feedback) tanto al estudiante como al profesor.

El profesor tendrá que determinar también el peso que representa la evaluación continua en la nota final. Dependiendo de qué se desee primar más, el proceso o el resultado de aprendizaje, variará el valor que se le asigne. Cuando se compagina la evaluación continua con la final, deberían valorarse ambas cosas: por un lado, cómo el estudiante ha progresado en la construcción de su conocimiento y en el desarrollo de las competencias y, por otro, el resultado final de este proceso. 
La evaluación sumativa o final se centra en el producto, certifica el resultado exigido del aprendizaje. Es el tipo de evaluación que se utiliza para calificar a los estudiantes al acabar una unidad o una asignatura con la finalidad de determinar el grado de logro de los objetivos previstos y valorar positiva o negativamente el producto evaluado. Con respecto a la evaluación continua existen tres diferencias fundamentales (Delgado et al. 2005):

- La evaluación sumativa no es formativa, no incide de manera directa en la mejora del proceso de aprendizaje de los estudiantes evaluados, precisamente por ser un tipo de evaluación que se realiza a posteriori, cuando el proceso se considera terminado.

- La evaluación sumativa otorga la puntuación al estudiante en relación con las calificaciones obtenidas por el resto de estudiantes. En cambio, en la evaluación continua la puntuación del estudiante está únicamente en relación con su propio proceso de aprendizaje.

- Los tipos de instrumentos para evaluar se diferencian también, en un examen final tradicional (evaluación sumativa) el tipo de preguntas que se suelen plantear son principalmente preguntas de desarrollo, resolución de casos prácticos, pruebas de elección múltiple o de verdadero y falso; mientras que los sistemas de evaluación continua optan por pruebas que se centran en experiencias de los alumnos, en debates, en el uso de carpetas de aprendizaje u otras actuaciones de los alumnos, es decir, se utilizan instrumentos de la llamada evaluación alternativa, métodos que facilitan la observación directa del trabajo de los estudiantes.

Si se toman en consideración las fases del proceso evaluativo, se pueden resumir los datos anteriormente expuestos como sigue:

\begin{tabular}{|l|l|}
\hline \multicolumn{1}{|c|}{ Fases del proceso evaluativo } & \multicolumn{1}{c|}{ Procedimientos } \\
\hline Identificación de objetivos & $\begin{array}{l}\text { Evaluación diagnóstica } \\
\text { Evaluación formativa } \\
\text { Evaluación sumativa }\end{array}$ \\
\hline Selección de instrumentos & $\begin{array}{l}\text { Pruebas objetivas } \\
\text { Pruebas alternativas }\end{array}$ \\
\hline Obtención y análisis de la información & $\begin{array}{l}\text { Verificación del grado de cumplimiento } \\
\text { de los criterios establecidos }\end{array}$ \\
\hline Resultados & Información (ev. diagnóstica) \\
& Retroinformación (ev.formativa) \\
& Calificación (ev. sumativa) \\
\hline
\end{tabular}

\subsection{La evaluación alternativa}

La evaluación alternativa pone el acento en métodos que facilitan la observación directa del trabajo de los estudiantes, al mismo tiempo que traspasan parte del control de la evaluación del profesor al estudiante. 
Las pruebas alternativas nacen inicialmente como reacción a la exclusividad de las pruebas objetivas y corroboran la necesidad de medir los resultados del aprendizaje desde prismas muy diferentes. Las razones de la búsqueda de otros métodos de evaluación, alejados de los tradicionales, las explica Mateo (en Mateo y Martínez 2008: 14):

Mediante la aplicación de las estrategias y de los instrumentos del enfoque tradicional, no sabemos con seguridad cómo están aprendiendo nuestros estudiantes y cómo les afecta la aplicación del currículum, más aún, el tipo de capacidades que ponen en juego nuestras pruebas no siempre están relacionadas con el tipo de conocimiento y habilidades que tienen verdadera relevancia en el mundo real.

Algunos de los rasgos que caracterizan la evaluación alternativa son:

- la utilización de otros instrumentos de evaluación (experimentos de los alumnos, proyectos, debates, carpetas de aprendizaje, productos de los estudiantes),

- la emisión de un juicio evaluativo basado en la observación y el (futuro) entorno profesional,

- la focalización de la evaluación en los alumnos,

- el retorno de la información sobre la evaluación y

- la participación del alumno en su propia evaluación.

El agente de la evaluación ya no es únicamente el profesor (heteroevaluación o evaluación por terceros), sino que los estudiantes pasan a ejercer un papel importante en su propia evaluación (autoevaluación) o la de sus compañeros (coevaluación o evaluación entre pares). Esto exige que el estudiante, como apuntábamos anteriormente, tenga información clara y precisa sobre los objetivos de aprendizaje, puesto que puede ser él quien tenga que determinar los criterios para evaluar.

\subsubsection{La carpeta de aprendizaje (CA)}

La CA tiene una doble función, puede servir como método de aprendizaje y como instrumento de evaluación. De entre los instrumentos de evaluación alternativa la CA (portafolio o dossier de aprendizaje) ${ }^{9}$ es el procedimiento más popular, constituye en el nuevo marco de créditos un sistema eficaz de gestión conjunta de los aprendizajes que está en la línea de la innovación docente y evaluativa que requieren los nuevos enfoques metodológicos. El uso de la CA supone (Blanch 2009: 4) cambios metodológicos (implementación de metodologías activas dentro y fuera del aula), cambios en la relación profesor/alumno (por la participación conjunta en la organización y gestión de la CA) y cambios en la estrategia de aprendizaje (para identificar, por ejemplo, las competencias específicas y transversales).

${ }^{9}$ Sobre la CA son interesantes los trabajos de Giné (2009), Berná (2008), Blanch (2009), Gregori (2009), Mateo y Martínez (2008) y Galán (2009) y los del Grupo GI-CAES. 
A través de la elaboración de una CA se pretende fundamentalmente que el alumno se motive e implique en su propio proceso de aprendizaje puesto que debe tomar decisiones sobre aspectos relevantes como por ejemplo el material que incluirá, los criterios para juzgar los materiales y las reflexiones que realizará sobre estos criterios. Se trata, en definitiva, de iniciar al estudiante en un tipo de aprendizaje basado en la autorreflexión lo cual favorecerá la implicación en la planificación de la propia formación. Para algunos autores, como Giné (2009: 7), los beneficios que aporta la elaboración de una CA para el estudiante son varios:

- ayuda a integrar el nuevo conocimiento en los conocimientos previos para construir nuevos modelos mentales (siguiendo el modelo constructivista del aprendizaje),

- favorece la comprensión y aplicación de conocimientos en vez de memorizar hechos,

- refleja la adquisición, por parte del alumno, de la capacidad de aplicar los conocimientos teóricos a situaciones reales,

- permite aprender de forma autónoma,

- facilita que el estudiante inicie su aprendizaje profesional desde el razonamiento reflexivo: el diseño de una CA y su redacción hace evidente la organización del pensamiento y el aprendizaje que se va realizando.

Martínez (en Mateo y Martínez 2008: 34) define la CA, en su terminología dossier de aprendizaje, como la "colección de materiales seleccionados con la intención de explicar el rendimiento o el aprendizaje realizado, reflexionar sobre esto y evaluarlo". Para Giné (2009: 7-8) una CA es

un conjunto estructurado de documentos (anotaciones, análisis, reflexiones, gráficas, etc.) elaborados por el estudiante, con la tutoría del docente, que se ordenan de forma cronológica o temática y que evidencia el progreso y el grado de cumplimiento de los objetivos planteados, también refleja las estrategias para la indagación, el pensamiento reflexivo, el rigor y el análisis.

La estructura de una CA puede ser cerrada (el docente indica el sumario), mixta (el docente indica los objetivos y el alumno establece el sumario) o abierta (se fijan partes obligatorias, pero el resto es libre), pero siempre gira en torno a tres ejes (Martínez, en Mateo y Martínez 2008: 32 y Berná 2008): la organización del contenido, la selección del material y la reflexión sobre las evidencias que aporta el material. Las partes de la CA y el contenido de cada una de ellas se recoge en la siguiente tabla: 


\begin{tabular}{|l|l|}
\hline \multicolumn{1}{|c|}{ Partes de la CA } & \multicolumn{1}{c|}{ Contenidos } \\
\hline Índice o mapa de la carpeta & \\
\hline Marco o contexto de aprendizaje & Breve descripción de la asignatura \\
\hline Objetivos de aprendizaje (competencias priorizadas) & Claramente definidos y priorizados \\
\hline Diario de actividades y tareas & Descripción breve de actividades y tareas \\
\hline Ejemplos de materiales (evidencias) & $\begin{array}{l}\text { Traducciones, documentación, fichas de análisis } \\
\text { textual, } \\
\text { fichas de consultas de diccionarios, textos } \\
\text { paralelos, } \\
\text { mapas conceptuales, resúmenes, reflexiones, } \\
\text { imágenes, artículos }\end{array}$ \\
\hline $\begin{array}{l}\text { Informe de autorreflexión, evidencias de } \\
\text { autoevaluación, } \\
\text { de reflexión (vid. el diario reflexivo en Bordas y } \\
\text { Cabrera 2001: 31 y ss.; Malick y Oster 2006) y su } \\
\text { comentario }\end{array}$ & $\begin{array}{l}\text { ¿Qtisfactorios), } \\
\text { ¿Qué aspectos no funcionaron todo lo bien que se } \\
\text { esperaba? (aspectos mejorables) } \\
\text { ¿Cómo cambiar estos últimos aspectos y por qué? } \\
\text { ¿Qué me falta por aprender? } \\
\text { Y ¿cómo lo puedo aprender? }\end{array}$ \\
\hline Objetivos futuros & \\
\hline
\end{tabular}

La evaluación de la CA ofrece varias posibilidades. Por un lado, se convierte en objeto de autoevaluación por parte del alumno mediante el informe de autorreflexión en el que debe dar respuesta a un protocolo de preguntas claramente definido. Por otro lado, es el medio de evaluación del proceso de aprendizaje por parte del profesor (heteroevaluación). También podría existir una tercera posibilidad, la coevaluación o evaluación inter pares en el caso, por ejemplo, de la evaluación entre compañeros. En todo caso, los criterios para evaluar una CA deben ser pertinentes y estar bien definidos (Berná 2008):

- presentación, estructuración y organización de la carpeta,

- relevancia de los temas seleccionados,

- reflexiones y profundización en las evidencias del proceso de aprendizaje,

- aportaciones personales y

- nivel de implicación y coherencia.

\section{Conclusiones}

La implementación de los nuevos planes de estudio, basados en el sistema de adquisición de competencias, requiere un cambio en los métodos de enseñar y en la relación profesor-alumno. La innovación docente supone introducir metodologías activas en el proceso de aprendizaje que requieren además métodos evaluativos innovadores que permitan valorar no sólo los conocimientos adquiridos sino también las demás cualidades implícitas en la noción de competencia, las habilidades y 
las actitudes. Es decir se deben evaluar saberes y conductas. Se evalúan procesos, no sólo resultados y evalúa el profesor, pero también el alumno, de esta forma la práctica de la autoevaluación se convierte en un método más de aprendizaje activo. Por medio de este método y la aplicación de otras estrategias como el ABP, el MC o AC se trata de que el alumno participe en su propia formación, sea protagonista de ésta de una manera responsable. Los tipos de evaluación tratados ofrecen posibilidades evaluativas pertinentes y coherentes con el resto de los elementos de la planificación docente. La evaluación actual no tiene por qué limitarse a la aplicación de un solo instrumento, las pruebas diagnósticas, formativas y sumativas representan toda una gama instrumental. La CA por su estructura y objetivos se adecuan especialmente a un marco pedagógico innovador al centrarse en la idea de aprender a aprender que conlleva la responsabilidad del alumno frente a determinadas decisiones que le acercan a lo que será su futuro profesional.

\section{Referencias bibliográficas}

Álvarez, I., «Evaluación del aprendizaje en la universidad», Revista electrónica de Investigación Psicoeducativa 14, 6 (1) (2008), 235-272.

Barkley, E., Cross, K., Howell, C., Técnicas de aprendizaje colaborativo. Madrid: Morata 2007.

Berná, J.D., «La carpeta de aprendizaje: una innovación docente en la asignatura de Radiología y Medicina Física Especial», Educación Médica 11, 4 (2008).

Biggs, J., Calidad del aprendizaje universitario. Madrid: Narcea 2005.

Blanch, S. et al., «Relaciones entre aprendizaje, cognición y tecnologías en la construcción del e-portafolio», Red U. Revista de Docencia Universitaria. Número monográfico III. Portafolios electrónicos y educación superior en España (2009). Consultado el 30 de mayo de 2010 en http://www.um.es//ead/Red_U/m3/.

Bordas, I. / Cabrera, F., «Estrategias de evaluación de los aprendizajes centrados en el proceso», Revista española de pedagogía LIX, 218 (2001), 25-48.

Delgado, A. $\mathrm{M}^{\mathrm{a}}$ et al., «Competencias y diseño de la evaluación continua y final en el Espacio Europeo de Educación Superior», en: Informe del proyecto I+D EA 2005-0054 (2005).

Echeverri, Á., «La importancia de la investigación colaborativa en la pedagogía de la traducción», Meta 50, 4 (2005).

Elena, P., «La crítica de la traducción: otros métodos, otros objetivos», Trans 3 (1998), 9-22.

Font, A., «Una experiencia de autoevaluación y evaluación negociada en un contexto de aprendizaje basado en problemas (ABP)», Revista de la Red Estatal de Docencia Universitaria 3, 2 (2003), 100-112.

Galán, A., La enseñanza de la traducción en la modalidad semipresencial. Tesis doctoral. Universidad Autónoma de Barcelona (2009).

Galán, A., «La enseñanza por competencias, por tareas y por objetivos de aprendizaje: el caso de la traducción jurídica portugués-español», Íkala. Revista de lenguaje y cultura 12,18 (2007), 27-56.

Giné, N. et al., Aplicación de la carpeta de aprendizaje en la universidad. Barcelona: Octaedro 2009. 
González Davies, M., Multiple Voices in the Classroom. Amsterdam: John Benjamins 2004. Gregori, E., «La carpeta de aprendizaje: qué, cómo y por qué», Observar 3 (2009), 55-88.

Hurtado Albir, A., Traducción y traductología. Introducción a la traductología. Madrid: Cátedra 2001.

Ibarra, S. y Rodríguez, G., Evaluación de competencias en Humanidades, Ciencias Sociales y Jurídicas. Curso IUCE. Universidad de Salamanca (2009).

Kelly, D., A Handbook for Translator Trainers. Manchester: St Jerome 2005.

Kiraly, D., «Project-Based Learning: A Case for Situated Translation», Meta 50, 4 (2005), 1098-1111.

Kiraly, D., A Social Constructivist Approach to Translator Education. Manchester: St Jerome 2000.

Lawick, H. van y Oster, U., «La evaluación de competencias transversales en la titulación de Traducción u Interpretación», en: Varela, $\mathrm{M}^{\mathrm{a}} \mathrm{J}$. (ed.), La evaluación en los estudios de traducción e interpretación. Sevilla: Bienza 2006, 69-93.

Le Boterf, G., Ingeniería de las competencias. Barcelona: Gedisa 2001.

López, B. e Hinojosa, E., Evaluación del aprendizaje. Alternativas y nuevos desarrollos. México: Editorial Trillas 2000.

Martínez Melis, N. y Hurtado Albir, A., «Assessment in Translation Studies: Research Needs», Meta 46, 2 (2001), 272-287.

Mateo, J. y Martínez Olmo, F., La evaluación alternativa de los aprendizajes. Barcelona: Octaedro 2008.

McDonald, R., «Nuevas perspectivas sobre la evaluación», Boletín Cinterfor 149, 2000, 4172.

Morales, P. y Landa, V., «Aprendizaje basado en problemas», Teoría 13 (2004), 145-157.

Nobs, $\mathrm{M}^{\mathrm{a}} \mathrm{L}$., Expectativas y evaluación en la traducción de folletos turísticos: estudio empírico con usuarios reales. Tesis doctoral. Universidad de Granada (2003).

Orozco, M. y Hurtado Albir, A., «Measuring Translation Competence Acquisition», Meta 47, 3 (2002), 375-402.

Orozco, M., «La evaluación diagnóstica, formativa y sumativa en la enseñanza de la traducción», en: Varela, $\mathrm{M}^{\mathrm{a}} \mathrm{J}$. (ed.). La evaluación en los estudios de traducción e interpretación. Sevilla: Bienza 2006, 47-67.

PACTE, «La competencia traductora y su adquisición», Quaderns 6 (2001), 39-45.

Parra, S., «Propuesta metodológica para la revisión de traducciones: principios generales y parámetros», Trans 11 (2007), 197-214.

Parra, S., La revisión de traducciones en la traductología: aproximación a la práctica de la revisión en el entorno profesional mediante el estudio de casos y propuestas de investigación. Tesis doctoral. Universidad de Granada (2005) http://hera.ugr.es/tesisugr/15472905.pdf

Ramírez, I., «Aprendizaje basado en problemas». Servicio de Innovación Educativa. Universidad Politécnica de Madrid (2005). Consultado el 2 de junio de 2010 en http:/www.mec.es/univ/proyectos2005/EA2005-0137.pdf

Rosenmund, A., «Konstruktive Evaluation: Versuch eines Evaluationskonzepts für den Unterricht», Meta 46, 2 (2001), 301-310.

Schippel, L. (Hg.), Übersetzungsqualität: Kritik-Kriterien-Bewertungshandeln. Berlin: Frank \& Timme 2006.

Tortadès, À., «Evaluación de la traducción en un entorno de aprendizaje colaborativo», en: Varela, M J J. (ed.), La evaluación en los estudios de traducción e interpretación. Sevilla: Bienza 2006, 93-112. 
Varela, M ${ }^{\mathrm{a}}$ J. y Postigo, E., «La evaluación en los estudios de traducción», Translator Education 9, 1 (2005).

Villardón, L., «Evaluación de aprendizaje para promover el desarrollo de competencias», Educatio siglo XXI, 24 (2006), 57-76.

Waddington, Ch., Estudio comparativo de diferentes métodos de evaluación de la traducción general (inglés-español). Tesis doctoral. Universidad Pontificia Comillas (2000).

Yániz, C., «Las competencias en el currículo universitario: implicaciones para diseñar el aprendizaje y para la formación del profesorado», Red U. Revista de Docencia Universitaria, número monográfico $1^{\circ}$ (2008). Consultado 5 de abril de 2010 en http://www.redu.m.es/Red_U/m1. 